\title{
The influence of surface energy on the self-cleaning of insect adhesive devices
}

\author{
M. J. Orchard ${ }^{1, \star}$, M. Kohonen ${ }^{2}$ and S. Humphries ${ }^{1}$ \\ ${ }^{1}$ Department of Biological Sciences, The University of Hull, Cottingham Road, Hull, East Yorkshire, HU6 7RX, UK and ${ }^{2}$ Department \\ of Quantum Science Physics Education Centre, Australian National University, Canberra, ACT 0200, Australia
}

*Author for correspondence (m.orchard@2004.hull.ac.uk)

Accepted 16 October 2011

\begin{abstract}
SUMMARY
The ability of insects to adhere to surfaces is facilitated by the use of adhesive organs found on the terminal leg segments. These adhesive pads are inherently 'tacky' and are expected to be subject to contamination by particulates, leading to loss of function. Here, we investigated the self-cleaning of ants and beetles by comparing the abilities of both hairy and smooth pad forms to selfclean on both high and low energy surfaces after being fouled with microspheres of two sizes and surface energies. We focused on the time taken to regain adhesive potential in unrestrained Hymenopterans (Polyrhachis dives and Myrmica scabrinodis) and Coccinellids (Harmonia axyridis and Adalia bipunctata) fouled with microspheres. We found that the reattainment of adhesion is influenced by particle type and size in Hymenopterans, with an interaction between the surface energy of the contaminating particle and substrate. In Coccinellids, reattainment of adhesion was only influenced by particle size and substrate properties. The adhesive organs of Coccinellids appear to possess superior self-cleaning abilities compared with those of Hymenopterans, although Hymenopterans exhibit better adhesion to both surface types.
\end{abstract}

Key words: adhesion, self-cleaning, insect ecology, frictional forces, free surface energy.

\section{INTRODUCTION}

Attachment devices are often used to counteract the pull of gravity. Animals use several types of attachment mechanism, from mechanical gripping using tarsal claws (Gladun et al., 2009) to specialised adhesive organs based on a variety of physical principles (Bullock et al., 2008; Drechsler and Federle, 2006). Insects use adhesive devices to enable foraging on a wide range of surfaces (Eigenbrode et al., 1999; Eigenbrode et al., 2000; Gorb et al., 2008) as well as for defence (Eisner and Aneshansley, 2000), mating (Bitar et al., 2009; Voigt et al., 2008) and oviposition (Bitar et al., 2009). While insect attachment devices include most known biological mechanisms, their adhesive organs are typically of two distinct types; smooth deformable organs (arolia) as found in Hymenoptera (ants, bees and wasps), or hairy adhesive pads as found in Coccinellids (ladybirds) (Bullock et al., 2008; Frantsevich and Gorb, 2004; Niederegger et al., 2002). Both pad types deform locally to allow adhesion to rough surfaces, and increase adhesive contact area through secretion of small amounts of fluid delivered between the pad and rough surface (Betz, 2003; Dirks et al., 2009; Persson, 2003; Votsch et al., 2002).

Insect attachment must allow for strong yet releasable adhesion to be made between the tarsal pads and both horizontal and inverted surfaces (Federle, 2006). Any loss of adhesive ability is likely to be detrimental in terms of loss of foraging capability and an increased risk of predation. To enable adhesion, insect feet are inherently 'tacky', employing an adhesive fluid secretion to maximise contact with a substrate (Gorb, 2005; Pohl and Beutel, 2004). The exact composition of the secretion is still unknown, although there is a general acknowledgement that it is an emulsion of lipophilic and hydrophilic components (Votsch et al., 2002), but no hydrophilic liquid has been identified (Geiselhardt et al., 2010). Indeed, Dirks and colleagues state that hydrophobic fluids stick well to both hydrophobic and hydrophilic surfaces (Dirks et al., 2009), and the inclusion of a hydrophilic component to the adhesive secretion used by insects has unclear benefits. Regardless of its composition, any secretion would only be of use on a rough surface where it would 'fill-in' the surface asperities to maximise contact area; however, because of the broken wax coverings of plant tissues and the fractal nature of weathered surfaces, it can be expected that all surfaces are to some extent rough. In systems using fluid in this way, contamination of the adhesive surfaces by loose material (contact contamination) can seriously compromise attachment ability (Clemente et al., 2010; Gorb and Gorb, 2006; Gorb et al., 2005). Contact contamination also reduces adhesive potential in biomimetic adhesive devices inspired by gecko feet (Lee and Fearing, 2008; Raibeck et al., 2008). The ability of insects to adhere to plant surfaces is also known to be dependent on surface properties (i.e. waxes, trichomes) (Eigenbrode, 2004; Eigenbrode et al., 1996; Eigenbrode et al., 1999; Eigenbrode et al., 2000; Gorb and Gorb, 2002; Gorb and Gorb, 2006; Lee and Fearing, 2008; Raibeck et al., 2008). Plant waxes in particular are known to cause problems for foraging insects (Eigenbrode, 2004; Markstadter et al., 2000; Riedel et al., 2003). The differing frictional coefficients of the wax surfaces alter the ability of insect foot secretions to wet plant surfaces (Gorb and Gorb, 2006; Wang et al., 2009). Frictional coefficients are used to describe the ratio of frictional force to normal load between two bodies, and are usually reported as static friction coefficients $\left(\mu_{\mathrm{s}}\right)$ for stationary objects and kinetic friction coefficients $\left(\mu_{\mathrm{k}}\right)$ for moving objects (Bowden and Tabor, 1950; Israelachvii, 2007). These coefficients of friction depend on the material's properties and the force(s) pressing them together. For example, dry glass on glass has a high static and kinetic coefficient of 0.94 and 0.4 , respectively, while dry PTFE on PTFE has $\mu_{\mathrm{s}}$ and $\mu_{\mathrm{k}}$ of 0.04 and 0.04 , respectively. Surfaces with high frictional coefficients generate greater frictional 
forces than those with low coefficients, i.e. glass on glass contact will generate more friction than PTFE on PTFE.

Some biological surfaces have been shown to exhibit selfcleaning properties; this self-cleaning of surfaces reduces or eliminates contact contamination. Given the prevalence and repercussions of contact contamination, self-cleaning attachment devices would appear to be a highly advantageous trait. Self-cleaning with water droplets is known for both plant (Barthlott et al., 1997; Ma and Hill, 2006; Solga et al., 2007) and animal surfaces (Baum et al., 2001; Baum et al., 2002; Clemente et al., 2009; Clemente et al., 2010; Genzer and Marmur, 2008; Gravish et al., 2010; Hansen and Autumn, 2005). However, until recently no adhesive organs were known to have self-cleaning capabilities (Hansen and Autumn, 2005). Models of the mechanical attachment of gecko setae suggest that they are able to self-clean through energetic disequilibrium between the adhesive forces attracting the contaminating particle to the adhesive setae and those attracting it towards the substrate (Autumn and Gravish, 2008). Essentially, bringing the fouled contact organ into contact with a surface that has a greater attraction to contaminating particles than the device leads to the removal of the particles from the adhesive organ. This self-cleaning ability has been suggested to be unique to the setal pad form of geckos and by extension those possessed by some iguanian and scincid species (Hansen and Autumn, 2005) that do not make use of a liquid secretion. It has also been suggested that the free surface energy [FSE, as defined by Israelachvii (Israelachvii, 2007)] of a substrate could alter the adhesive forces generated in the 'dry' adhesive system found in geckos (Autumn and Peattie, 2002). FSE also determines the spreading or 'wetting' of a liquid over a substrate; surfaces with a low FSE, such as Fluon ${ }^{\circledR}$ for example, cause liquid to bead up. In general, high frictional coefficients are found for surfaces with a high FSE and vice versa (Rabinowicz, 1961; Israelachvii, 2007). Recent work has suggested that the 'dry' adhesive ability of gecko toe pads is influenced by relative humidity via the formation of capillary bridges between the spatulae and the substrate (Huber et al., 2005), and softening of the $\beta$-keratin of the spatulae, allowing more intimate contact with the substrate (Puthoff et al., 2010), or enhanced by the use of an adhesive secretion (Hsu et al., 2011). Hui and colleagues suggest that particle rolling, where small movements of the adhesive setae push the contaminating particles to the outer edge of the adhesive pad, may also contribute to the self-cleaning of the hairy pad structures in geckos (Hui et al., 2006). A similar model for self-cleaning of hairy pads in insects has been put forward by Clemente and colleagues (Clemente et al., 2010).

In the only study of insect adhesive pad self-cleaning to date (Clemente et al., 2010), it was suggested that the self-cleaning mechanisms proposed for the dry fibrillar adhesive pads of geckos could be logically extended to cover the setal pad forms of insects that use an adhesive secretion. Here, we present a comparison of the self-cleaning abilities of the two main insect adhesive pad types. We compared the ability of insects with different types of adhesive pad fouled with particles differing in their FSE to escape over vertical walls also with differing FSE values. We used two differently sized species of Coccinellidae, the harlequin ladybird [Harmonia axyridis (Pallas 1773)] and the two-spot ladybird [Adalia bipunctata (Linnaeus 1758)], which have hairy pads, along with two differently sized species of ant, the Asian weaver ant (Polyrhachis dives, Smith 1857) and the red elbowed Spanish ant (Myrmica scabrinodis, Nylander 1846), which have smooth pads, to also determine what affect the physical size of an insect has on the self-cleaning ability of each pad type. We hypothesised that the physical size of the contaminating particle would influence the recovery of adhesion, and that such cleaning would depend on the FSE of both the contaminating particles and the substrate, with surfaces with high FSE (glass) predicted to be better cleaning surfaces that those with low values of FSE, as it is reasonable to assume that surfaces with high FSE values would generate sufficient shear forces to remove contaminating particles from the pad through friction, compared with those with low FSE.

For the purposes of this study, the time it takes an insect to recover adhesive ability by 'scrabbling' on the vertical walls, after its adhesive pads have been fouled, was defined as successful selfcleaning. Self-cleaning by shear forces would be facilitated by the higher frictional force between the contaminating particle and the substrate than the adhesive forces holding the contaminating particle to the pad. We show that there is an interaction between the physical properties of the contaminating particle and the substrate that significantly influences the self-cleaning ability of insect adhesive pads.

\section{MATERIALS AND METHODS}

Specimens of $P$. dives and $M$. scabrinodis were ordered from a supplier (http://www.antstore.net/shop); two colonies of each species were housed in glass formicaria $(19 \times 21 \times 19 \mathrm{~cm})$. Specimens of $A$. bipunctata were obtained from biocontrol suppliers (http://www.greengardener.co.uk), while specimens of $H$. axyridis were collected locally from a public park in Kingston upon Hull (latitude $53^{\circ} 45^{\prime} 31^{\prime \prime} \mathrm{N}, 0^{\circ} 21^{\prime} 11^{\prime \prime} \mathrm{W}$ ); both were housed in glass formicaria $(16 \times 20 \times 6 \mathrm{~cm})$. Insects were maintained as lab stocks; ants were fed a mix of chopped mealworms and honey, while ladybirds were fed a prepared artificial food source (Majerus and Kearns, 1989) ad libitum three times a week. Measurements of insect mass, length and maximal pad contact area were taken for each species and are presented in Table 1: masses were derived by weighing two series of 10 insects and are presented as mean values; length measurements were determined from optical images taken using a Canon PowerShot S31S digital camera (www.canon.co.uk), attached to a Nikon SMZ800 stereo-microscope (www.nikoninstruments.com); contact area was calculated from scanning electron microscope (SEM) micrographs of each insect species with the maximal length and width of the pads recorded for each insect using packages in ImageJ (http://rsbweb.nih.gov/ij/).

To test the effects of pad type on self-cleaning after contamination, two series of cylindrical vials $\left(\mathrm{SiO}_{2}, 75 \times 25 \mathrm{~mm}\right)$ were prepared; although the vial walls are curved, their radius of curvature is several orders of magnitude greater than that of the individual adhesive pads

Table 1. Mean mass, body length and adhesive pad contact area for each species

\begin{tabular}{lcccc}
\hline Species & Mass $(\mathrm{mg})$ & Length $(\mathrm{mm})$ & Pad type & Contact area $\left(\mu \mathrm{m}^{2}\right)$ \\
\hline Adalia bipunctata & $12 \pm 0.6$ & $4 \pm 2$ & Hairy & $33.29( \pm 0.25) \times 10^{3}$ \\
Harmonia axyridis & $35 \pm 1.0$ & $9 \pm 2$ & Hairy & $114.60( \pm 1.17) \times 10^{3}$ \\
Myrmica scabrinodis & $5 \pm 0.2$ & $5 \pm 2$ & Smooth & $3.07( \pm 0.06) \times 10^{3}$ \\
Polyrhachis dives & $15 \pm 0.3$ & $8 \pm 1$ & Smooth & $40.65( \pm 0.39) \times 10^{3}$ \\
\hline
\end{tabular}

Adhesive pad contact area is mean pad area across all legs. Mean values are presented \pm s.e.m. ( $N=20$ individuals per species). 


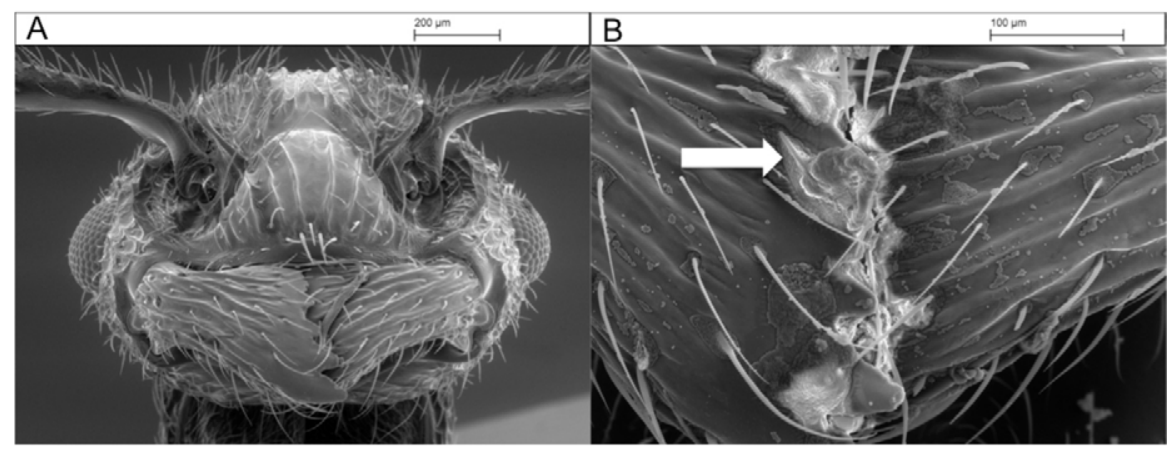

Fig. 1. Scanning electron microscope (SEM) micrographs of (A) Myrmica scabrinodis and (B) Polyrhachis dives jaws post-preening after contamination of their adhesive pads with glass and PTFE particles, respectively. Post-preening contamination of jaws was not found for ants fouled with glass spheres but was observed for those insects fouled with PTFE particles. To emphasize the contamination by PTFE particles, Fig. 1B is shown at a higher magnification and particle contamination is indicated with an arrow.

of the insects, and therefore can be considered a flat surface. While we cannot rule out a minor bracing effect of paired legs, we consider the additional frictional forces to be minimal given the vial curvature. All vials were cleaned by sonication in a hexane bath (HPLC grade, $95 \% n$-hexane; Fisher Scientific, Loughborough, UK) for 1 min then left to stand for $2 \mathrm{~min}$ and rinsed with isopropanol (HPLC grade iso-propyl alcohol, 99\% pure; Fisher Scientific). After cleaning, one series was left untreated ('clean glass': high FSE, mean contact angle of $3 \pm 2 \mathrm{deg}$ ), while the other was treated with DCDMS (dichlorodimethylsilane, $\geq 98.5 \%$ pure; Sigma-Aldrich, http://www.sigmaaldrich.com) in a vapour phase similar to that used by West and colleagues (West et al., 2007) ('treated glass': low FSE, mean contact angle $105 \pm 3 \mathrm{deg}$ ) to produce a low energy surface comparable to plant substrates [Heliconia denisflora $28.4 \pm 4.3 \mathrm{deg}$ and Brassica oleracea $160.3 \pm 0.8 \mathrm{deg}$ (Barthlott et al., 1997)]. All vials were rinsed with hexane and blown dry with pure nitrogen before each replicate to ensure the surfaces were free from environmental contaminants such as dust. Contact angles of experimental surfaces were measured each day $(N=10)$ for the duration of the study by placing $5 \mu \mathrm{l}$ of MilliQ purified water onto the surface and directly recording the contact angle using a Krüss DSA-10 (Alton, UK) drop shape analysis machine. This enabled us to verify that the surface treatments did not deteriorate over the course of the study.

\section{Contamination}

Unlike previous mechanistic studies (Clemente et al., 2010) where the insects were restrained and artificially fouled with particles, in this study insects were allowed to free roam within an environment that would cause contamination in a more 'natural' manner. Insects were fouled with either glass or PTFE microspheres by being placed into a Teflon dish with a dusting of particles on its inner surfaces for $2 \mathrm{~min}$. Two size classes of microsphere were used: 'small' (glass, $19 \pm 8 \mu \mathrm{m}$ mean \pm s.d. diameter, VWR-Jencons, uk.vwr.com); PTFE, $21 \pm 13 \mu \mathrm{m}$ mean \pm s.d. diameter, Sigma-Aldrich) and 'large' (glass, $111 \pm 24 \mu \mathrm{m}$ mean \pm s.d. diameter; PTFE, $123 \pm 60 \mu \mathrm{m}$, mean \pm s.d. diameter). After contamination, the insects were transferred to one of the two series of vials and left unperturbed under video observation. For small insect species (M. scabrinodis, A. bipunctata) large particle sizes were not tested, as particle diameter was greater than the claw width, making them unlikely to foul (M.J.O., personal observation).

\section{Self-cleaning}

All experiments, including control tests, were carried out in multiples of 20 replicates (total number of replicates in study; $P$. dives $N=200$, M. scabrinodis $N=120, H$. axyridis $N=200$ and $A$. bipunctata $N=120)$ at laboratory relative humidity $(28-34 \%)$ and temperature $\left(20-24^{\circ} \mathrm{C}\right)$. Six vials were lined up in parallel and continuous video recordings were taken for $30 \mathrm{~min}\left(8 \mathrm{frames} \mathrm{s}^{-1}\right)$ using a webcam (Logitech Quickcam ${ }^{\circledR}$ Pro, www.logitech.com) controlled by HANDYAVI (version 4.2; Anderson's AZcendant software, www.azcendant.com). Although the image resolution was not sufficient to view the adhesive devices in the large array, several analogous replicates were undertaken wherein the focal area of the camera was refined to show the adhesive devices more clearly.

The time taken to adhere ( $\left.t_{\text {adhere }}\right)$ to the inner vertical wall of the glass vial was taken from the video footage. Insects were judged to have adhered when all six legs were on the vertical wall of the vial and the insect was able to move freely. The measured $t_{\text {adhere }}$ was adjusted to exclude periods during which the insect was not moving, under the assumption that there would be no effective shear or inplane forces to shed contaminating particles. If an insect failed to adhere to the vial wall within $30 \mathrm{~min}$ the trial was awarded a censored value. This censored value was used to determine the number of insects that adhered to the inner vial walls within the $30 \mathrm{~min}$ period and analysed using survival analysis [see Crawley (Crawley, 2010) for further details on survival analysis]. Additionally, time spent preening $\left(t_{\text {preen }}\right)$ was noted from the same video files to allow comparison of active cleaning within each experimental group and between species, and compared using ANOVA and a post hoc Tukey test. It has been noted that reduced friction triggers grooming behaviour in beetles (Hosoda et al., 2010), so comparison of the time spent preening allowed a measure of the duration of contamination of the adhesive devices and as such an indication of the reduced friction between the adhesive device and the substrate.

All statistical analyses were carried out using $\mathrm{R}$ version 2.10.1 (R Development Core Team, 2009). Survival analysis was used to investigate the relationship between (1) the physical size of the particle and the insect's ability to escape and (2) the physical

Table2. Mean ( \pm s.e.m.) $t_{\text {preen }}$ for large species, with different particle type, size and substrate combinations

\begin{tabular}{|c|c|c|c|c|c|c|c|c|}
\hline Species & sg/utv & sPTFE/utv & Ig/utv & IPTFE/utv & $\mathrm{sg} / \mathrm{tv}$ & sPTFE/tv & $\lg / \mathrm{tv}$ & IPTFE/tv \\
\hline Harmonia axyridis & $709 \pm 175$ & $119 \pm 108$ & $28 \pm 12$ & $39 \pm 31$ & $1030 \pm 168$ & $4 \pm 3$ & $84 \pm 47$ & $395 \pm 4$ \\
\hline Polyrhachis dives & $872 \pm 8$ & $278 \pm 168$ & $189 \pm 0.20$ & $428 \pm 171$ & $1702 \pm 22$ & $106 \pm 95$ & $146 \pm 118$ & $414 \pm 167$ \\
\hline
\end{tabular}

$t_{\text {preen, time spent preening (in s). }}$

Column headings refer to particle-substrate combination. sg, small glass particles; sPTFE, small PTFE particles; Ig, large glass particles; IPTFE, large PTFE particles; utv, untreated glass vial; tv, treated glass vial. 
Table 3. Mean ( \pm s.e.m.) $t_{\text {preen }}$ for small species, with different particle type, size and substrate combinations

\begin{tabular}{lcccc}
\hline Species & sg/utv & sPTFE/utv & sg/tv & sPTFE/tv \\
\hline Adalia bipunctata & $1065 \pm 135$ & $982 \pm 192$ & $510 \pm 212$ & $456 \pm 192$ \\
Myrmica scabrinodis & $1239 \pm 203$ & $473 \pm 187$ & $171 \pm 133$ & $1239 \pm 118$ \\
\hline
\end{tabular}

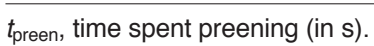

Column headings refer to particle-substrate combination. sg, small glass particles; sPTFE, small PTFE particles; Ig, large glass particles; IPTFE, large PTFE particles; utv, untreated glass vial; tv, treated glass vial.

properties of the substrate-particle interaction and the insect's ability to escape. Survival analysis was used as our data show right censoring (Crawley, 2010). Survival analysis is used where the data show censoring; that is, if at the end of the study the event of interest has not occurred (in this case, if the ant had not managed to adhere). As it is impossible to say that the individual would never adhere it is instead expected that the individual will adhere at an unknown time in the future and the data are said to be censored. Survival analysis allows one to make statistical comparisons between censored data sets where standard statistics would suffer bias as a result of missing data (Crawley, 2010; Heagerty, 2005; Heagerty et al., 2000). We used a linear model of the form $t_{\text {adhere }}=$ particle type $\times$ particle size $\times$ container type for large insects, while for small insects the particle size term was removed. Data were modelled using both an exponential distribution (which assumes constant hazard) and a Weibull distribution (which allows for nonconstant hazard with time). ANOVA indicated that the Weibull distribution was the better fit to our data and those results are presented here. However, use of exponential distribution models does not qualitatively alter our conclusions. Non-significant terms were sequentially removed based on Akaike information criterion (AIC) values, leaving only parameters with a significant influence on the output of the model.

\section{RESULTS}

Effect of preening time on self-cleaning

Several of the insects were observed to preen themselves with their mouthparts during the experiments, and individuals that displayed high levels of preening behaviour were collected post-experiment and imaged using scanning electron microscopy (Zeiss Evo 600.2 SEM; www.zeiss.co.uk). Micrographs of the insects showed clean jaws for those individuals contaminated with glass particles (Fig. 1A), while those contaminated with PTFE particles displayed contamination of the mouth parts (Fig. 1B). The duration of $t_{\text {preen }}$ was found to differ significantly between species $(t=2.8954$, d.f. $=328.97, P=0.004)$, although there was no significant difference between the large insects $(t=-0.1433$, d.f. $=195.748, P=0.88)$ in $t_{\text {preen }}$ for either particle or substrate type. Both $A$. bipunctata and $M$. scabrinodis spent significantly more time preening than the larger insects $(t=5.065$, d.f. $=129.352, P<0.001)$. Mean duration $( \pm$ s.e.m. $)$ of preening behaviour is shown in Table 2 for the large insects while the data for small insects are presented in Table 3.

\section{Survivorship curves for controls of $t_{\text {adhere }}$}

To provide a baseline of adhesive ability for each species, control experiments were conducted in which an unfouled insect was placed in a representative vial of each type and $t_{\text {adhere }}$ recorded (Fig. 2). To control for confounding factors such as pad size and behavioural differences, we analysed the data first by species. In a further analysis, trials were grouped by the physical size of the insects, large ( $P$. dives and $H$. axyridis) and small ( $M$. scabrinodis and $A$. bipunctata), to determine whether self-cleaning ability is a function of physical factors such as the size of the attachment pad. The resulting survivorship curves for large species, with different particle and substrate combinations are presented in Fig. 3, and those for the small species are presented in Fig. 4.

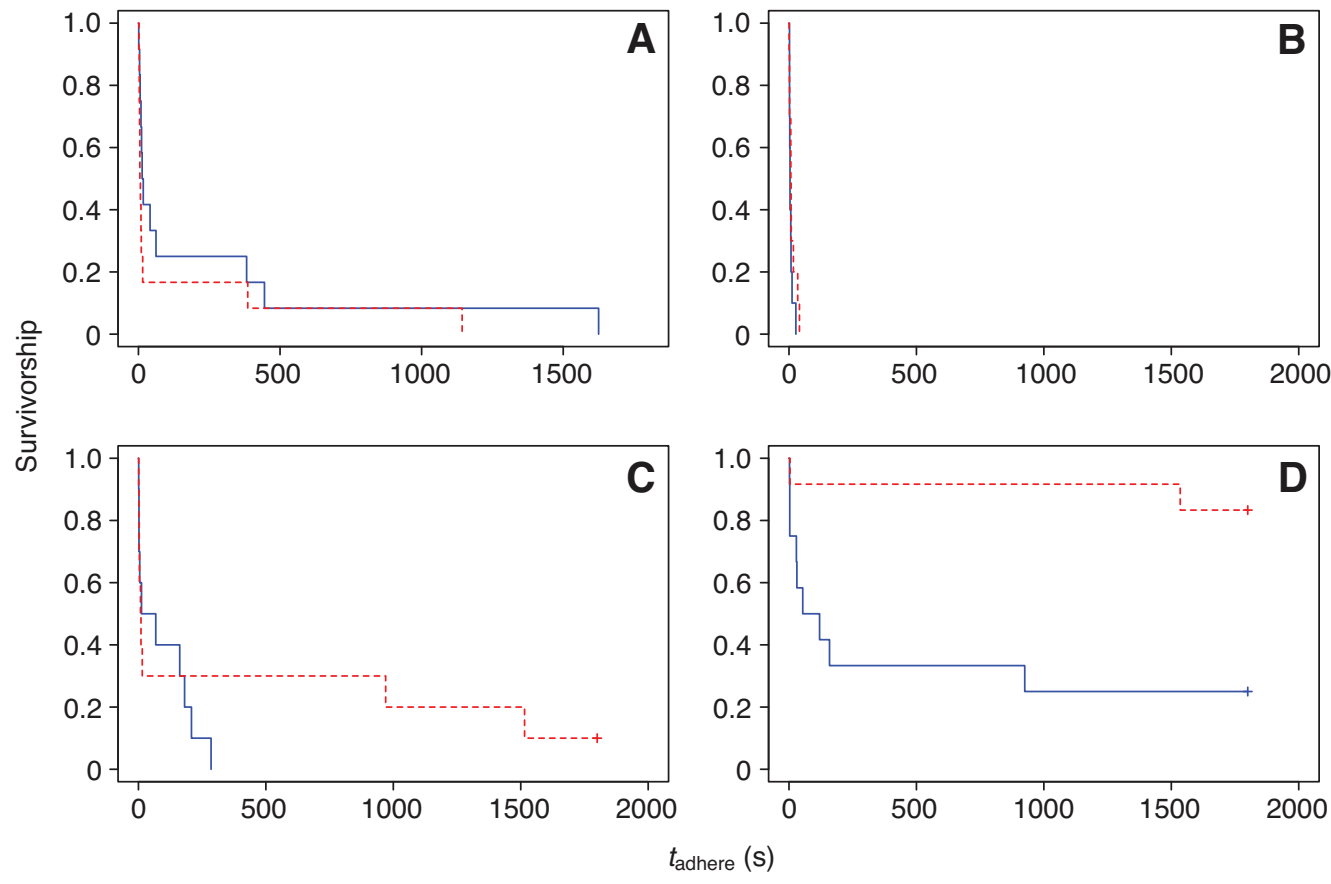

Fig. 2. Survivorship curves of time taken to adhere ( $\left.t_{\text {adhere }}\right)$ controls for (A) Harmonia axyridis, (B) P. dives, (C) Adalia bipunctata and (D) $M$. scabrinodis. For all plots, solid blue lines are untreated vials, dashed red lines are treated vials $(N=20$ for each species-vial combination). A step in the curve indicates an insect was successful in adhering to the inner vial wall, ' + ' at the end of the line indicates there were still insects that had failed to adhere by the end of the $30 \mathrm{~min}$ time period and a line crossing the $x$ axis indicates all insects successfully adhered within the time period. 

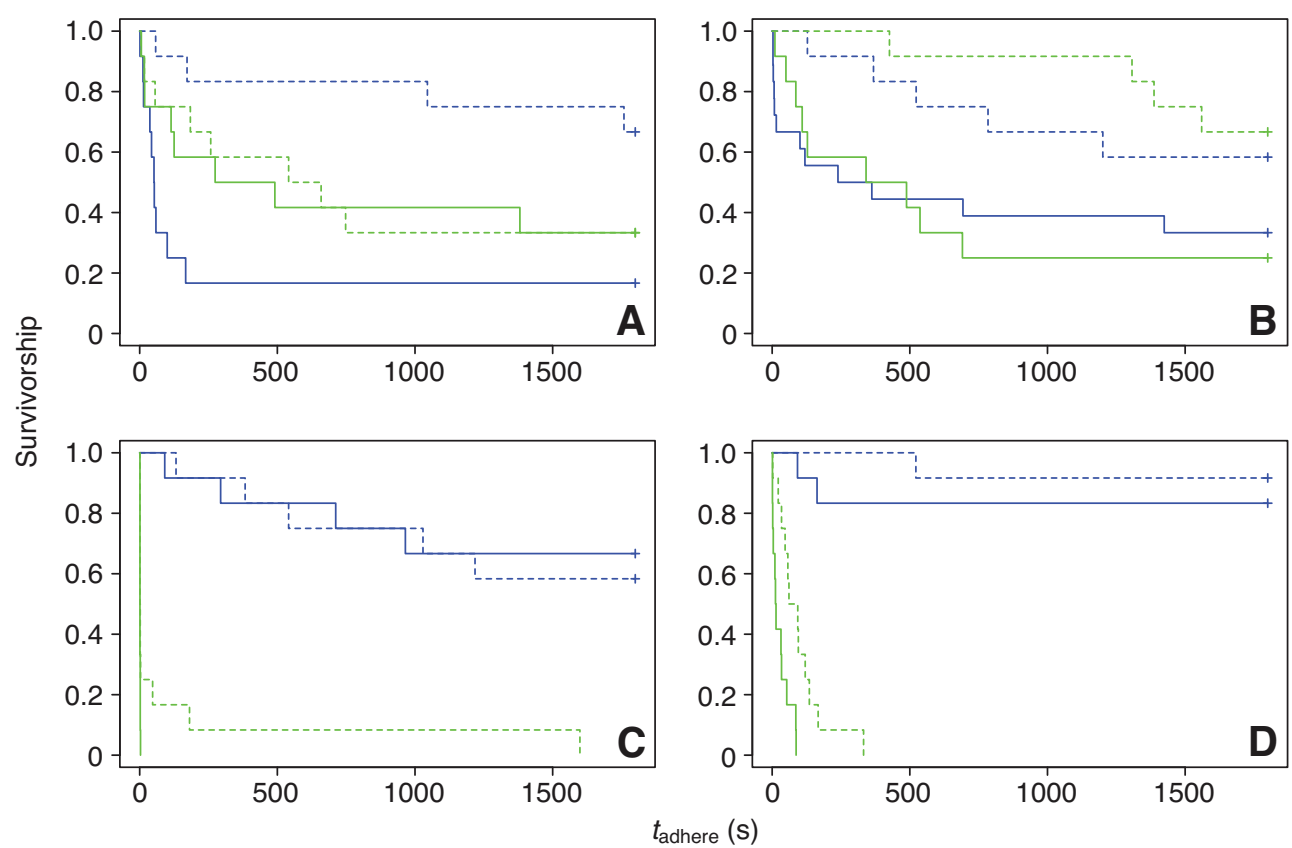

Fig. 3. Survivorship curves for $t_{\text {adhere }}$ in experimental treatments with large insects and large particles (A, H. axyridis; D, $P$. dives) or small particles (B, $H$. axyridis; $\mathrm{C}$, $P$. dives). For all plots, blue lines are PTFE particles, green lines are glass particles, continuous lines are untreated vials and dashed lines are treated vials $(N=20$ for each combination for each species). A step in the curve indicates an insect was successful in adhering to the inner vial wall, ' + ' at the end of the line indicates there were still insects that had failed to adhere by the end of the $30 \mathrm{~min}$ time period and a line crossing the $x$-axis indicates all insects successfully adhered within the time period.
There was no significant difference between times taken to adhere to the control surfaces for large insects either within (harlequin ladybird $H$. axyridis, d.f. $=1, F=2.66, P=0.10$, Fig. 2A; weaver ant $P$. dives, d.f. $=1, F=1.60, P=0.22$, Fig. $2 \mathrm{~B}$ ) or between (d.f. $=1, F=2.66$, $P=0.109)$ species. No significant difference between $t_{\text {adhere }}$ to the control surfaces was found for $A$. bipunctata (d.f. $=1, F=5.94, P=0.23$, Fig. 2C); however, there was a significant difference in $t_{\text {adhere }}$ for M. scabrinodis (d.f. $=1, F=5.94, P=0.02$, Fig. $2 D$ ), with a longer time taken to adhere to the treated vials. A difference in $t_{\text {adhere was found }}$ between small species (d.f. $=1, F=4.15, P=0.04$ ), with $A$. bipunctata adhering in a faster time to both surface types compared with $M$. scabrinodis, possibly as a result of the smaller contact area of the adhesive pad of $M$. scabrinodis (Table 1). No significant difference was found for time to adhere between large and small insects when compared between species (d.f. $=3, F=1.25, P=0.29$ ).

\section{Effect of FSE and pad morphology on regaining adhesive ability}

When the data were grouped according to pad type, a significant effect of both particle (d.f. $=1, Z=6.55, P<0.001$ ) and substrate (d.f.=1, $Z=2.37, P<0.001)$ FSE was found for smooth pads. We found that insects contaminated with small PTFE particles took longer to adhere to either surface type, but there was no interaction between the two terms (d.f. $=2$, log-likelihood $=-470.1, P>0.001$ ). For hairy pads, only the FSE of the substrate had a significant influence on time taken to escape (d.f. $=1$, log-likelihood $=-637.5, P<0.001$ ). Irrespective of particle size or type, more time was required to adhere to the treated vial surfaces.

\section{Interactions between particle type, size and substrate on the regaining of adhesive ability}

As can be observed from Figs 3 and 4 and Tables 4 and 5, for interactions between surfaces with a high frictional coefficient, glass on glass offered better self-cleaning than glass on treated surfaces regardless of particle size, and it was found that PTFE was harder to remove on all surfaces for both pad types.

We found a significant three-way interaction for $t_{\text {adhere }}$ between particle size, particle FSE and substrate FSE for $H$. axyridis (d.f.=7, log-likelihood $=-458, P<0.001)$. In $P$. dives, $t_{\text {adhere }}$ was significantly greater for small particles than for large ones (d.f. $=1, Z=3.758$, $P<0.001$ ), with a significant trend for individuals contaminated with particles of low frictional values taking longer to adhere than those contaminated with particles with high FSE values (d.f. $=1, Z=0.57$, $P<0.001$ ), regardless of substrate properties. While an influence of
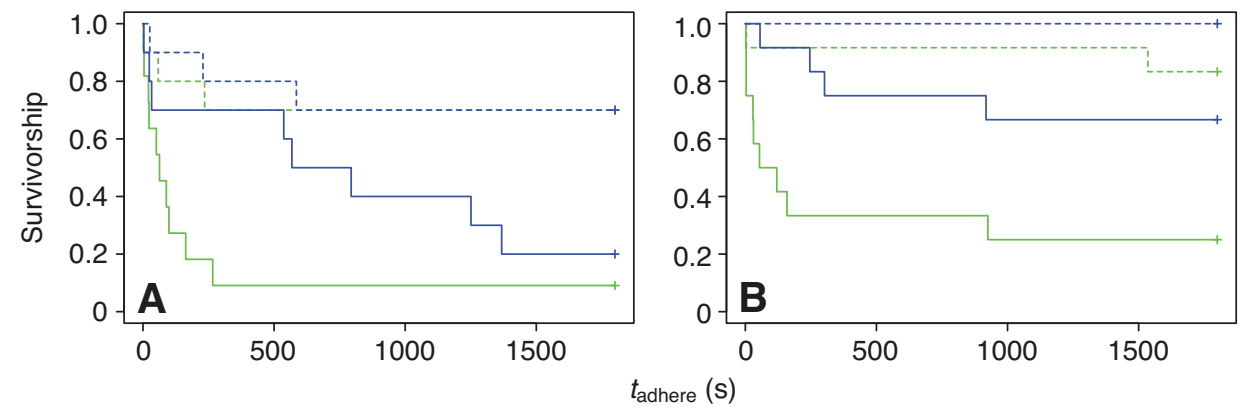

Fig. 4. Survivorship curves for $t_{\text {adhere }}$ in experimental treatments with small insects (A, A. bipunctata; B, M. scabrinodis). For both plots, blue lines are PTFE particles, green lines are glass particles, continuous lines are untreated vials and dashed lines are treated vials $(N=20$ for each combination for each species). A step in the curve indicates an insect was successful in adhering to the inner vial wall, ' + ' at the end of the line indicates there were still insects

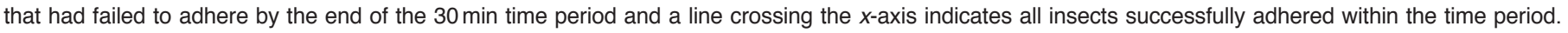


Table 4. Mean ( \pm s.e.m.) $t_{\text {adhere }}$ values for large insects for all particle and substrate combinations

\begin{tabular}{lcccccccc}
\hline Species & sg/utv & sPTFE/utv & Ig/utv & IPTFE/utv & sg/tv & sPTFE/tv & Ig/tv & IPTFE/tv \\
\hline Harmonia axyridis & $653 \pm 146$ & $765 \pm 171$ & $782 \pm 179$ & $344 \pm 77$ & $1590 \pm 355$ & $1338 \pm 299$ & $805 \pm 180$ & $1452 \pm 324$ \\
Polyrhachis dives & $28 \pm 6$ & $1521 \pm 340$ & $1.4 \pm 0.3$ & $1371 \pm 306$ & $97.08 \pm 21$ & $1693 \pm 378$ & $21.9 \pm 4.0$ & $1203 \pm 378$ \\
\hline
\end{tabular}

$t_{\text {adhere, }}$ time to adhere (in s).

Because of the number of insects that did not manage to adhere within the 30 min time period the mean values are skewed. sg, small glass particles; sPTFE, small PTFE particles; Ig, large glass particles; IPTFE, large PTFE particles; utv, untreated glass vial; tv, treated glass vial.

substrate treatment was found to significantly affect $t_{\text {adhere, }}$ with longer $t_{\text {adhere }}$ for the treated vial walls regardless of contaminating particle size or type (d.f. $=1, Z=0.40, P<0.001$ ), there was also a significant interaction between particle type and vial treatment in $P$. dives, with those insects contaminated with PTFE particles taking significantly longer to adhere to treated vial walls (d.f. $=4$, loglikelihood=-311.5, $P<0.001$ ) (see Fig. 3C,D). The A. bipunctata results indicated a significant effect of the FSE of the contaminating particle (d.f.=2, log-likelihood $=-172.7, P<0.001$ ), with a nonsignificant trend observed for substrate type $(P=0.092)$, although this was discarded using our AIC criteria; it can be seen from Fig. 4A that insects contaminated with glass or PTFE particles showed a similar survival curve on the treated substrate. For M. scabrinodis, $t_{\text {adhere }}$ was influenced by both particle and substrate FSE but no interaction between the two (d.f. $=2$, log-likelihood $=-113.2$, $P>0.001$ ) was found.

\section{DISCUSSION}

While a self-cleaning ability has been documented in a number of animal taxa [geckos (Hansen and Autumn, 2005), stick insects and dock beetles (Clemente et al., 2010)] and hypothesised more generally (Federle, 2006), the mechanics of self-cleaning in wet adhesive systems have been unclear. Here, for the first time we were able to examine self-cleaning in freely walking, rather than tethered (Clemente et al., 2010) insects. We were able to partially disentangle the interacting effects of FSE and pad type in the insect wet adhesion system. The results from the fouled insects show that although both hairy and smooth pad morphologies are capable of self-cleaning there are subtle differences between them. Adhesive pad types differed in both their self-cleaning ability (the number of contaminating particles removed) and the rate of recovery of adhesion once fouled (time taken to adhere to the vial walls), but these effects varied with the interplay of surface and particle FSE. Although it is true that while trapped in the vial the rear legs of the insect may have become re-contaminated with particles shed from the front limbs, this in itself is comparable to 'natural' events whereby the insect would have to 'clean' its feet while constantly being exposed to recontamination from plant waxes and environmental detritus; thus, the level of recontamination is likely to be small.

\section{Effect of preening time on self-cleaning}

While $t_{\text {preen }}$, i.e. physically removing the contaminating particles from the insect's body and adhesive organs, did not differ between species of the same size, small insects were found to spend a longer time preening than larger ones (Tables 2,3$)(t=5.065$, d.f. $=129.352$, $P<0.001$ ), possibly because of the relative size difference between small pads and particles compared with large pads and particles making it harder to scrabble at the inner vial walls. In order to assess the level of contamination of the jaws (a proxy for preening activity), a subset of insects used in the experiments were examined using a SEM. Fig. 1 shows examples of these images, providing visual confirmation of differences in the level of contamination between particle types.

\section{Effect of FSE and pad morphology on regaining adhesive ability}

While both pad types experienced loss of adhesive potential when fouled with microspheres, smaller particles were harder for both pad types to shed regardless of the physical size of the insect. The reported interaction between the FSE of the substrate and contaminating particles can be observed in Figs 3 and 4, where $t_{\text {adhere }}$ was significantly longer for insects on treated glass surfaces contaminated with PTFE particles regardless of particle size, pad morphology or insect mass. This resulted in a higher number of insects trapped at the end of the study. The ability to self-clean in ladybirds (Coccinellids) was strongly influenced by the difficulty of adhering to surfaces with a low FSE, with the difference in time taken to adhere to the surfaces being dependent on particle size for $H$. axyridis, and on particle type for $A$. bipunctata. The ability to regain adhesion in both Hymenoptera (ants: smooth pads) was influenced by particle type and size, with an interaction between the frictional coefficient of the contaminating particle and substrate. The results of this study lend partial support to results previously reported by Clemente and colleagues (Clemente et al., 2010), where an effect of particle size was found for the regaining of adhesion in the dock beetle Gastrophysa viridula, with better recovery of adhesion for beetles than for the stick insect Carausius morosus when contaminated with large $(45 \mu \mathrm{m})$ and small $(1 \mu \mathrm{m})$ particles, but not for particles of $10 \mu \mathrm{m}$. We suggest that the difference found in the present study may be due to the differing morphologies of the pads, with smaller particles becoming trapped within the setal hairs of the hairy pads in a similar way to that reported by Clemente and colleagues (Clemente et al., 2010).

The dense setae of the hairy pad type may simply allow easier shedding of contaminating particles than for smooth pads through normal forces (acting perpendicular to the surface) as suggested by previous studies (Clemente et al., 2010; Hui et al., 2006; Hansen and Autumn, 2005), or through a rolling action (Hui et al., 2006)

Table 5. Mean ( \pm s.e.m.) $t_{\text {adhere }}$ values for small insects for all particle and substrate combinations

\begin{tabular}{lcccc}
\hline Species & sg/utv & sPTFE/utv & sg/tv & sPTFE/tv \\
\hline Adalia bipunctata & $234 \pm 52$ & $817 \pm 112$ & $1432 \pm 134$ & $1344 \pm 115$ \\
Myrmica scabrinodis & $560 \pm 125$ & $1326 \pm 118$ & $1628 \pm 149$ & $1521 \pm 147$ \\
\hline
\end{tabular}

$t_{\text {adhere, }}$ time to adhere (in s).

Because of the number of insects that did not manage to adhere within the $30 \mathrm{~min}$ time period the mean values are skewed. sg, small glass particles; sPTFE, small PTFE particles; utv, untreated glass vial; tv, treated glass vial. 


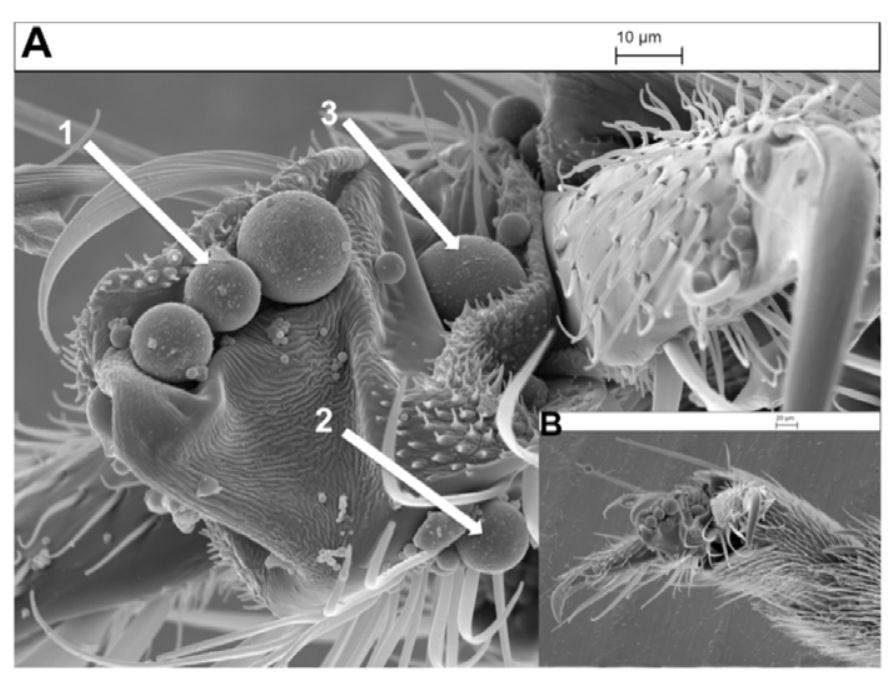

Fig. 5. (A) High magnification SEM micrograph of the arolium of $P$. dives with $19 \pm 8 \mu \mathrm{m}$ glass particles ensnared within the folds of the arolium. The outer edges of the arolium are comparatively clean while glass spheres can be seen held around the rolled distal edges (arrows 1 and 2) as well as within the folds adjacent to the tarsal claws (arrow 3). (B) The placement of the arolium within the tarsal claws of $P$. dives can be observed in this lower magnification image. Scale bar in B is $20 \mu \mathrm{m}$.

where small lateral movements of the setae may facilitate cleaning via rolling the particle off the pad. Using this rolling argument, Clemente and colleagues (Clemente et al., 2010) suggested that the lack of setae on smooth pads can lead to particles becoming embedded within the folds of the arolium. This at first seems counterintuitive as if the pads are better able to clean through shear forces then why would particles become trapped within the folds of the arolium? To explain this we put forward the following hypothesis; whilst the arolium is fully deployed and at its maximum extension, particles would be rolled off the surface through contact with the substrate; however, once the arolium has started to reduce in size as the insect releases contact with the substrate (between steps), any particles still on the surface of the arolium would become trapped because of the folding mechanism of the arolium as it is retained between the tarsal claws (Federle et al., 2001). We find support for this hypothesis in SEM images of the adhesive devices of ants that failed to self-clean within this study (Fig. 5). It is also possible that although the arolium is less able to remove contaminants through application of normal forces it may be better able to self-clean through shear forces, which would be supported by the scrabbling and foot-dragging behaviour insects were seen to perform during this study (M.J.O., unpublished data).

\section{Interactions of particle type, size and substrate on the regaining of adhesive ability}

Although the glass particles used in this and previous studies are functionally spherical, the PTFE particles had a greater range of shapes and may have been better able to be packed into gaps between setae or in folds of the arolium. It seems likely that packing behaviour influenced the ability of the pads to remove PTFE particles compared with glass particles.

The weak ability of the hairy pads to adhere to the low energy substrate is interesting, as previous studies have suggested that the morphology of the hairy pads have been optimised for surface attachment (Federle, 2006). It is possible that on surfaces with a low FSE the adhesive secretion may bead up, leaving it unable to wet the surface adequately, so reducing capillary adhesion, although this would be in contrast to current theory regarding the emulsion hypothesis and its benefits for attachment to both hydrophobic and hydrophilic surfaces.

Furthermore, when contaminated with PTFE particles $P$. dives individuals were observed to drag their rear legs behind their body, leaving behind a smear of fluid on the vial wall within which several contaminating particles were trapped. While this dragging behaviour was absent in the other species, both the Coccinellids used in this study were occasionally observed to remain stationary for long periods of time, and upon moving again left behind yellow-coloured liquid deposits on the substrate that were 'flushed' from the adhesive pad, and within which were trapped the contaminating particles; however, it should be noted that both the dragging and 'flushing' behaviours observed were displayed infrequently and no insect exhibiting such behaviours was used in the subsequent analysis. Although larger animals need not move faster by stepping more frequently, but may simply have a longer stride, the larger insects in this study did tend to move faster and to step more often than the smaller species (M.J.O., personal observation). This may have had an additive effect on the rate of adhesive recovery postcontamination; a possible explanation for this is if both a large and a small insect were trapped in adjacent vials but were constantly moving they would have a different degree of movement, with the larger insect moving more frequently and having an increased efficacy of the adhesive pads. This may explain why the larger insects preened less but regained adhesive ability faster.

We conclude that while the self-cleaning rate of hairy and smooth pads in free-roaming insects is similar, interactions between the relative FSEs of the contaminating particle and the substrate only had a significant effect on the hairy pad form, suggesting that the mechanisms used by the two pad types do differ, and that this difference in ability has a morphological basis. The extent to which these interactions influence the behaviour and life history traits of insects is open for further work. The results of our study suggest that it is impractical to generalise about adhesive capabilities across species or even between differing size classes within a species.

\section{ACKNOWLEDGEMENTS}

The authors wish to thank the two anonymous referees for helpful comments on the manuscript, Mr M. Anyon for helpful discussion on the nature of friction and $\mathrm{Mr}$ Tony Sinclair for his work obtaining the SEM images.

\section{FUNDING}

The University of Hull 80th anniversary scholarship scheme provided funding for the research carried out within this paper.

\section{REFERENCES}

Autumn, K. and Gravish, N. (2008). Gecko adhesion: evolutionary nanotechnology. Philos. Trans. R. Soc. Lond. A 366, 1575-1590.

Autumn, K. and Peattie, A. M. (2002). Mechanisms of adhesion in geckos. Int. Comp. Biol. 42, 1081-1090.

Bathlott, W. and Neinhuis, C. (1997). Purity of the scared lotus, or escape from contamination in biological surfaces. Planta 202, 1-8.

Baum, C., Meyer, W., Roessner, D., Siebers, D. and Fleischer, L, G. (2001). A zymogel enhances the self-cleaning abilities of the skin of the pilot whale (Globicephala melas). Comp. Biochem. Physiol. 130A, 835-847.

Baum, C., Meyer, W., Stelzer, R., Fleischer, L. G. and Siebers, D. (2002). Average nanorough skin surface of the pilot whale (Globicephala melas, Delphinidae): considerations on the self-cleaning abilities based on nanoroughness. Mar. Biol. 140, 653-657.

Betz, O. (2003). Structure of the tarsi in some Stenus species (Coleoptera, Staphylinidae): external morphology, ultrastructure, and tarsal secretion. J. Morphol. 255, 24-43.

Bitar, L. A., Voigt, D., Zebitz, C. P. W. and Gorb, S. N. (2009). Tarsal morphology and attachment ability of the codling moth Cydia pomonella L. (Lepidoptera, Tortricidae) to smooth surfaces, J. Insect Physiol. 55, 1029-1038. 
Bowden, F. and Tabor, D. (1950). The Friction and Lubrication of Solids, Part 1. Oxford: Oxford University Press.

Bullock, J. M. R., Drechsler, P. and Federle, W. (2008). Comparison of smooth and hairy attachment pads in insects: friction, adhesion and mechanisms for directiondependence. J. Exp. Biol. 211, 3333-3343.

Clemente, C. J., Beale, A., Bullock, J. M. and Federle, W. (2009). Evidence for selfcleaning in fluid-based smooth and hairy adhesive systems of insects. Comp. Biochem. Physiol. 153A, S114-S133.

Clemente, C. J., Bullock, J. M. R., Beale, A. and Federle, W. (2010). Evidence for self-cleaning in fluid-based smooth and hairy adhesive systems of insects. J. Exp. Biol. 213, 635-642.

Crawley, M. J. (2010). The R Book. Chichester: John Wiley and Sons Ltd.

Dirks, J. H., Clemente, C. J. and Federle, W. (2009). Insect tricks: two-phasic foot pad secretion prevents slipping. J. R. Soc. Interface 7, 587-593.

Drechsler, P. and Federle, W. (2006). Biomechanics of smooth adhesive pads in insects: influence of tarsal secretion on attachment performance. J. Comp. Physiol. 192A, 1213-1222.

Eigenbrode, S. D. (2004). The effects of plant epicuticular waxy blooms on attachment and effectiveness of predatory insects. Arthropod Struct. Dev. 33, 91-102.

Eigenbrode, S. D., Castagnola, T., Roux, M. B. and Steljes, L. (1996). Mobility of three generalist predators is greater on cabbage with glossy leaf wax than on cabbage with a wax bloom. Entomol. Exp. Appl. 81, 335-343.

Eigenbrode, S. D., Kabalo, N. N. and Stoner, K. A. (1999). Predation, behaviour, and attachment by Chrysoperla plorabunda larvae on Brassica oleracea with different surface waxblooms. Entomol. Exp. Appl. 90, 225-235.

Eigenbrode, S. D., Rayor, L., Chow, J. and Latty, P. (2000). Effects of wax bloom variation in Brassica oleracea on foraging by a vespid wasp. Entomol. Exp. Appl. 97 161-166.

Eisner, T. and Aneshansley, D. J. (2000). Defence by foot adhesion in a beetle (Hemisphaerota cyanea). Proc. Natl. Acad. Sci. USA 97, 6568-6573.

Federle, W. (2006). Why are so many adhesive pads hairy? J. Exp. Biol. 209, 26112621.

Federle, W., Brainerd, E. I., McMahon, T. A. and Holldobler, B. (2001)

Biomechanics of the movable pretarsal adhesive organ in ants and bees. Proc. Natl. Acad. Sci. USA 98, 6215-6220.

Frantsevich, L. and Gorb, S. (2004). Structure and mechanics of the tarsal chain in the hornet, Vespa crabro (Hymenoptera: Vespidae): implications on the attachment mechanism. Arthropod Struct. Dev. 33, 77-89.

Geiselhardt, S. F., Lamm, S., Gack, C. and Peschke, K. (2010). Interaction of the liquid epicuticular hydrocarbon and tarsal adhesive secretion in Leptinotarsa decemlineata Say (Coleoptera: Chrysomelidae). J. Comp. Physiol. A 196, 369-378.

Genzer, J. and Marmur, A. (2008). Biological and synthetic self-cleaning surfaces. Mater. Res. Bull. 33, 742-746.

Gladun, D., Gorb, S. N. and Frantsevich, L. I. (2009). Alternative tasks of the insect arolium with special reference to Hymenoptera. In Functional Surfaces in Biology, Vol. 2, Adhesion Related Phenomena (ed. S. N. Gorb), p. 67-104. Dordrecht: Springer.

Gorb, E. V. and Gorb, S. N. (2002). Attachment ability of the beetle Chrysolina fastuosa on various plant surfaces. Entomol. Exp. Appl. 105, 13-28.

Gorb, E. V. and Gorb, S. N. (2006). Physiochemical properties of functional surfaces in pitchers of the carnivorous plant Nepenthes alata Blanco (Nepenthaceae). Plant Biol. 8, 841-848.

Gorb, E. V., Haas, K., Henrich, A., Enders, S., Barbakadaze, N. and Gorb, S. N. (2005). Composite structure of the crystalline epicuticular wax layer of the slippery zone in the pitchers of the carnivorous plant Nepenthes alata and its effect on insect attachment. J. Exp. Biol. 208, 4651-4662.

Gorb, E. V., Voight, D., Eigenbrode, S. D. and Gorb, S. N. (2008). Attachment force of the beetle Cryptolaemus montrouzieri (Coleoptera, Coccinellidae) on leaflet surfaces of mutants of the pea Pisum sativum (Fabaceae) with regular and reduced wax coverage. Arthropod Plant Interact. 2, 247-259.

Gorb, S. N. (2005). Uncovering insect stickiness: structure and properties of hairy attachment devices. Am. Entomol. 51, 31-35.
Gravish, N., Wilkinson, M., Sponberg, S., Parness, A., Esparza, N., Soto, D., Yamaguchi, T., Broide, M., Cutosky, M., Creton, C. et al. (2010). Rate-dependent frictional adhesion in natural and synthetic gecko setae. J. R. Soc. Interface 7, 259269.

Hansen, W. R. and Autumn, K. (2005). Evidence for self-cleaning in gecko setae. Proc. Natl. Acad. Sci. USA 102, 385-389.

Heagerty, P. J. (2005). Survival model predictive accuracy and ROC curves. Biomimetics 61, 92-105.

Heagerty, P. J., Lumley, T. and Pepe, M. S. (2000). Time-dependent ROC curves for censored survival data and a diagnostic marker. Biomimetics 56, 337-344.

Hsu, P. Y., Ge, L., Li, X., Stark, A. Y., Wesdemiotis, C., Niewiarowski, P. H. and Dhinojwala, A. (2011). Direct evidence of phospholipids in gecko footprints and spatula-substrate contact interface detected using surface-sensitive spectroscopy. J. R. Soc. Int., doi:10.1098/rsif.2011.0370.

Israelachvii, J. (2007). Intermolecular and Surface Forces, 3rd edn. San Diego, CA: Academic Press.

Lee, J. and Fearing, R. S. (2008). Contact self-cleaning of synthetic gecko adhesive from polymer microfibers. Langmuir 24, 10587-10591.

Ma, M. and Hill, M. (2006). Superhydrophobic surfaces, Curr. Opin. Colloid Interface Sci. 11, 193-202.

Majerus, M. and Kearns, P. (1989). Ladybirds, Naturalist Handbook Vol. 10. Dorset: The Richmond Publishing Co. Ltd.

Markstadter, C., Federle, W., Jetter, R., Riederer, R. and Holldobler, B. (2000). Chemical composition of the slippery epicuticular wax blooms on Macaranga (Euphorbiaceae) ant-plants, Chemoecology 10, 33-40.

Niederegger, S., Gorb, S. and Jiao, Y. (2002). Contact behaviour of tennent setae in attachment pads of the blowfly Calliphora vicina (Diptera, Calliphoridae). J. Comp. Physiol. A 187, 961-970.

Persson, B. N. J. (2003). On the mechanism of adhesion in biological systems. J. Chem. Phys. 118, 7614-7621.

Pohl, H. and Beutel, R. G. (2004). Fine structure of adhesive devices of Strepsiptera (Insecta). Arthropod Struct. Dev. 33, 31-43.

Puthoff, J., Prowse, M. S., Wilkinson, M. and Autumn, K. (2010). Changes in materials properties explain the effects of humidity on gecko adhesion. J. Exp. Biol. 213, 3699-3704.

Rabinowicz, E. (1961). Influence of surface energy on friction and wear phenomena. J. Appl. Phys. 32, 1440-1444.

Railbeck, L., Reap, J. and Bras, B. (2008). Investigating environmental benefits of biologically inspired self-cleaning surfaces. 15th CIRP International Conference on Life Cycle Engineering. Paris: The International Academy for Production Engineering.

Riedel, M., Eichner, A. and Jetter, R. (2003). Slippery surfaces of carnivorous plants: composition of epicuticular wax crystals in Nepenthes alata Blanco pitchers. Planta 218, 87-97.

Smith, J. M., Barnes, W. J. P., Downie, J. R. and Ruxton, G. D. (2006). Structural correlates of increased adhesive efficiency with adult size in the toe pads of hylid tree frogs. J. Comp. Physiol. A 192, 1193-1204.

Solga, A., Cerman, Z., Striffler, B. F., Spaeth, M. and Barthlott, W. (2007). The dream of staying clean: lotus and biomimetic surfaces. Bioinspir. Biomim. 2, S126S134.

Voigt, D., Schuppert, J. M., Dattinger, S. and Gorb, S. N. (2008). Sexual dimorphism in the attachment ability of the Colorado potato beetle Leptinotarsa decemlineata (Coleoptera: Chrysomelidae) to rough substrates. J. Insect Physiol. 54, 765-776.

Votsch, W., Nicholson, G., Muller, R., Stierhof, Y. D., Gorb, S. and Schwarz, U. (2002). Chemical composition of the attachment pad secretion of the locust Locusta migratoria. Insect Biochem. Mol. Biol. 32, 1605-1613.

Wang, L., Zhou, Q., Zheng, Y. and Xu, S. (2009). Composite structure and properties of the pitcher surface of the carnivorous plant Nepenthes and its influence on the insect attachment system. Prog. Nat. Sci. 19, 1657-1664. 